\title{
On randomly colouring locally sparse graphs
}

\author{
Alan Frieze and Juan Veralli \\ Department of Mathematics \\ Carnegie Mellon University \\ Pittsburgh PA15213, USA \\ a lanerandom.math. cmu.edu \\ jveradandrew.cmu.edu
}

received Aug 30, 2004, accepted Apr 13, 2006.

\begin{abstract}
We consider the problem of generating a random $q$-colouring of a graph $G=(V, E)$. We consider the simple Glauber Dynamics chain. We show that if for all $v \in V$ the average degree of the subgraph $H_{v}$ induced by the neighbours of $v \in V$ is $\ll \Delta$ where $\Delta$ is the maximum degree and $\Delta>c_{1} \ln n$ then for sufficiently large $c_{1}$, this chain mixes rapidly provided $q / \Delta>\alpha$, where $\alpha \approx 1.763$ is the root of $\alpha=e^{1 / \alpha}$. For this class of graphs, which includes planar graphs, triangle free graphs and random graphs $G_{n, p}$ with $p \ll 1$, this beats the $11 \Delta / 6$ bound of Vigoda [20] for general graphs.
\end{abstract}

Keywords: Counting Colourings, Sampling, Markov Chains

\section{Introduction}

Markov Chain Monte Carlo (MCMC) is an important tool in sampling from complex distributions. It has been successfully applied in several areas of Computer Science, most notably volume computation [3], [15], [16] and estimating the permanent of a non-negative matrix [12]. It was used by Jerrum [10] to generate a random $q$-colouring of a graph $G$, provided $q>2 \Delta$. This has led to the challenging problem of determining the smallest value of $q$ for which it is possible to generate a (near)-uniform sample from the set $\mathcal{Q}$ of proper $q$-colourings of $G$ in polynomial time. We cannot expect the chain to mix for $q \leq \Delta+1$ and at present it is unknown as to whether or not it mixes rapidly for say $q=\Delta+2$. Vigoda [20] improved Jerrum's result by reducing the lower bound on $q$ to $11 \Delta / 6$. This is still the best lower bound on $q$ for general graphs.

The lack of complete success on the general problem has led to the analysis of restricted classes of graphs. Suppose that we consider Glauber dynamics on the set $\mathcal{Q}$. Specifically we will consider the heat bath dynamics, which may be described as follows. We start from an arbitrary proper $q$-colouring $X_{0} \in \mathcal{Q}$. At step $t>0$ of the process, in state $X_{t-1} \in \mathcal{Q}$, we choose a vertex $v_{t} \in V$ uniformly

\footnotetext{
${ }^{\dagger}$ Research supported by NSF grant CCR-0200945.

1365-8050 @ 2006 Discrete Mathematics and Theoretical Computer Science (DMTCS), Nancy, France
} 
at random. Then we choose $j_{t}$ uniformly at random from the colours with which $v_{t}$ may be properly coloured, given $X_{t-1}\left(V \backslash v_{t}\right)$. We recolour $v_{t}$ with $j_{t}$ to give $X_{t} \in \mathcal{Q}$.

Dyer and Frieze [2] considered this process restricted to the class of graphs $\mathcal{G}\left(c_{1}, c_{2}\right)$ : the set of graphs with $n$ vertices, maximum degree $\Delta \geq c_{1} \log n$ and girth $g \geq c_{2} \log \Delta$. They showed using the idea of "burn-in" that for $c_{1}, c_{2}$ sufficiently large, Glauber Dynamics mixed in $O(n \log n)$ time, provided $q>\alpha \Delta$ where $\alpha \approx 1.763$ is the root of $\alpha=e^{1 / \alpha}$. Molloy [17] improved this result by reducing the lower bound on $q$ to being more than $\beta \Delta$ where $\beta \approx 1.489$ is the root of $\left(1-e^{-1 / \beta}\right)^{2}+\beta e^{-1 / \beta}=1$. The girth asumptions were then relaxed by Hayes [7] to $g \geq 5$ for $k / \Delta>\alpha$ and $g \geq 6$ for $k / \Delta>\beta$. Subsequently, Hayes and Vigoda [8] made considerable progress, using a non-Markovian coupling, and reduced the lower bound on $k / \Delta$ to $(1+\epsilon)$ for all $\epsilon>0$, which is nearly optimal. Their result requires girth $g \geq 9$. However, the large maximum degree restriction remained. This was replaced by $\Delta \geq \Delta_{0}$ in Dyer, Frieze, Hayes and Vigoda [5], with the same restrictions on girth as in [7]. Dyer, Flaxman, Frieze and Vigoda [4] show that for sparse random graphs, the number of colours required for rapid mixing is of order the average rather than maximum degree whp. Goldberg, Martin and Paterson [6] prove results on the related notion of strong spatial mixing.

In this paper we avoid girth restrictions and consider locally sparse graphs instead. We say that a graph $G=(V, E)$ is $\gamma$-locally sparse if for all $v \in V$, the average degree of the graph induced by the neighbourhood $N(v)$ is at most $\gamma$. Thus planar graphs are always 6-locally-sparse and triangle free graphs are 0-locally-sparse.

Theorem 1.1 Suppose that $q \geq(\alpha+\epsilon) \Delta$ where $\epsilon$ is a small positive constant. Let $G$ be an $n$-vertex $\gamma$-locally sparse graph with $\gamma \leq \epsilon^{2} \Delta / 10$ and $\Delta \geq c_{1} \log n$. If $c_{1}=c_{1}(\epsilon)$ is sufficiently large then the Glauber dynamics converges to within variation distance $e^{-1}$ from uniform over $\mathcal{Q}$ in at most $O(n \ln n)$.

Notice that if $G=G_{n, p}$ and $\frac{c_{1} \log n}{n} \leq p \leq \epsilon^{2} / 11$ then whp $G$ satisfies the conditions of the theorem. Note also that the chromatic number of a triangle-free graph is $O(\Delta / \log \Delta)$ - see Johansson [14] or Molloy and Reed [18] or Alon, Krivelevich and Sudakov [1] or Vu [21].

Our proof uses coupling and relies on a recent idea from Hayes and Vigoda [9] that utilises the fact that we can couple against the steady state distribution of the chain. Note that the theorem generalises Theorem 4 of [9].

In what follows we will assume that $n$ is sufficiently large and $\epsilon$ is sufficiently small to satisfy our inequalities.

\section{Preliminaries}

We will consider two copies of Glauber Dynamics, $\left(X_{t}, t \geq 0\right)$ and $\left(Y_{t}, t \geq 0\right)$. Here $X_{0}$ is an arbitrary colouring and $Y_{0}$ is chosen from the uniform (stationary) distribution over $\mathcal{Q}$. At time $t$, the Hamming distance between $X_{t}, Y_{t}$ is defined by

$$
H\left(X_{t}, Y_{t}\right)=\sum_{v \in V} 1_{X_{t}(v) \neq Y_{t}(v)} .
$$

We will couple the two processes as in Jerrum [10]. Here $v_{t}$ is the same in both processes and then the choice of colours is maximally coupled. For vertex $w$ let

$$
A\left(X_{t}, w\right)=\left\{c \in[q]: c \notin X_{t}(N(w))\right\}
$$


be the set of colours available to colour $w$ in $X_{t}$ if $v_{t}=w$.

Let $a\left(X_{t}, w\right)=\left|A\left(X_{t}, w\right)\right|$ and define the terms $A\left(Y_{t}, w\right), a\left(Y_{t}, w\right)$ analogously.

It is shown in [9] that

$$
\mathbf{E}\left(H\left(X_{t+1}, Y_{t+1}\right)-H\left(X_{t}, Y_{t}\right)\right) \leq-\frac{1}{n} H\left(X_{t}, Y_{t}\right)+\frac{1}{n} \sum_{w \in V} \frac{\left|\left\{u \in N(w): X_{t}(u) \neq Y_{t}(u)\right\}\right|}{\max \left\{a\left(X_{t}, w\right), a\left(Y_{t}, w\right)\right\}} .
$$

We will show that for $w \in V$ and $\delta=\epsilon / 10$,

$$
\operatorname{Pr}\left(a\left(Y_{t}, w\right) \leq \Delta /(1-\delta)\right) \leq n^{-4} .
$$

Assuming that $a\left(Y_{t}, w\right) \geq \Delta /(1-\delta)$ in (1) we get

$$
\begin{aligned}
\mathbf{E}\left(H\left(X_{t+1}, Y_{t+1}\right)-H\left(X_{t}, Y_{t}\right)\right) & \leq-\frac{1}{n} H\left(X_{t}, Y_{t}\right)+\frac{1}{n} \frac{H\left(X_{t}, Y_{t}\right) \Delta}{\Delta /(1-\delta)} \\
& \leq-\frac{\delta}{n} H\left(X_{t}, Y_{t}\right) .
\end{aligned}
$$

So conditional on an event of probability $1-O\left(n^{-3}\right)$, we have

$$
\mathbf{E}\left(H\left(X_{t+1}, Y_{t+1}\right) \mid X_{t}, Y_{t}\right) \leq\left(1-\frac{\delta}{n}\right) H\left(X_{t}, Y_{t}\right)
$$

Thus if $T=n(1+\ln n) \delta^{-1}$ then conditional on an event of probability $1-O\left(n^{-2} \log n\right)$, we have

$$
\mathbf{E}\left(H\left(X_{T}, Y_{T}\right)\right) \leq e^{-1}
$$

and so unconditionally

$$
\mathbf{E}\left(H\left(X_{T}, Y_{T}\right)\right) \leq e^{-1}+o(1) .
$$

Hence the mixing time of the Glauber Dynamics is $O(n \ln n)$ as claimed.

\section{Bounding the number of available colours}

Fix $v \in W$ and let $H_{v}$ be the subgraph of $G$ induced by $N(v)$. Let $B(v)$ be the vertices of $N(v)$ that have degree at least $\gamma \delta^{-1}$ in $H_{v}$. Note that $\gamma \delta^{-1} \leq \epsilon \Delta$ and

$$
|B(v)| \leq \delta|N(v)|
$$

since $G$ is $\gamma$-locally-sparse.

Let

$$
N^{*}(v)=N(v) \backslash B(v)=\left\{w_{1}, w_{2}, \ldots, w_{d}\right\} .
$$

Now let let us fix the colours $\kappa(v)$ used at

$$
v \in W_{v}=V \backslash N^{*}(v) .
$$

Let us use the term allowable for colorings of $N^{*}(v)$ which respect this conditioning. Let $\Omega$ be the set of allowable colourings of $N^{*}(v)$. 
Let $a^{*}(\sigma, v)$ be the number of colours not used on $N^{*}(v)$. Note that (3) implies

$$
a(\sigma, v) \geq a^{*}(\sigma, v)-\delta|N(v)| .
$$

Now consider the following process $\mathcal{P}_{\sigma}$ for producing an allowable colouring of $H_{v}$. Here $\sigma \in \Omega$. We let $\sigma_{0}=\sigma$ and for $j=1,2, \ldots, d$ let $\sigma_{j}$ be obtained from $\sigma_{j-1}$ as follows: Keep $\sigma_{j}\left(w_{k}\right)=\sigma_{j-1}\left(w_{k}\right)$ for $k \neq j$ and choose $\sigma_{j}\left(w_{j}\right)$ randomly from what is available to it.

Let $Z_{\sigma}$ be the number of colours not appearing on a vertex in $N^{*}(v)$ if we start with $\sigma_{0}=\sigma$.

Lemma 3.1 If $\sigma$ is chosen uniformly from $\Omega$ then for any $c>0$,

$$
\operatorname{Pr}\left(a^{*}(\sigma, v) \geq c\right)=\operatorname{Pr}\left(Z_{\sigma} \geq c\right) .
$$

Proof We first prove that

$$
\text { If } \sigma_{0} \text { is chosen uniformly from } \Omega \text { then } \sigma_{d} \text { is also uniform over } \Omega \text {. }
$$

We do this by induction on $j$, with base case $j=0$.

$$
\begin{aligned}
\operatorname{Pr}\left(\sigma_{j}=\sigma\right) & =\sum_{\sigma^{\prime} \in \Omega} \operatorname{Pr}\left(\sigma_{j}=\sigma \mid \sigma_{j-1}=\sigma^{\prime}\right) \operatorname{Pr}\left(\sigma_{j-1}=\sigma^{\prime}\right) \\
& =\frac{1}{|\Omega|} \sum_{\sigma^{\prime} \sim \sigma} \operatorname{Pr}\left(\sigma_{j}=\sigma \mid \sigma_{j-1}=\sigma^{\prime}\right)
\end{aligned}
$$

Here $\sigma^{\prime} \sim \sigma$ if $\sigma, \sigma^{\prime}$ differ only at $w_{j}$.

$$
\begin{aligned}
& =\frac{1}{|\Omega|} \sum_{\sigma^{\prime} \sim \sigma} \frac{1}{\left|\left\{\sigma^{\prime}: \sigma^{\prime} \sim \sigma\right\}\right|} \\
& =\frac{1}{|\Omega|} .
\end{aligned}
$$

Now $a^{*}\left(\sigma_{d}, v\right)=Z_{\sigma_{0}}$ and so

$$
\operatorname{Pr}\left(a^{*}\left(\sigma_{d}, v\right) \geq c\right)=\operatorname{Pr}\left(Z_{\sigma_{0}} \geq c\right)
$$

and the lemma follows from (5)

For $w \in N^{*}(v)$ let

$$
L(w)=[q] \backslash\left\{\kappa(u): u \in N(w) \backslash N^{*}(v)\right\}
$$

be the colours not specifically barred from $w$ by the current conditioning. Then let

$$
L^{*}\left(w_{j}\right)=[q] \backslash\left\{\sigma_{j-1}(u): u \neq w_{j}\right\} \quad \text { for } j=1,2, \ldots, d
$$

be the colours available to $w_{j}$ when it is re-coloured by $\sigma_{j}$. 
We will first estimate the (conditional) expectation of $Z_{\sigma}$ for arbitrary $\sigma$. Suppose that $x \in[q]$. Let $\theta_{x, j}=1_{x \in L\left(w_{j}\right)}$ and let $\theta_{x, j}^{*}=1_{x \in L^{*}\left(w_{j}\right)}$. Then we have

$$
\begin{aligned}
\operatorname{Pr}\left(x \notin \sigma_{d}\left(N^{*}(v)\right)\right) & =\prod_{j=1}^{d} \operatorname{Pr}\left(\sigma_{d}\left(w_{j}\right) \neq x \mid \sigma_{d}\left(w_{i}\right) \neq x, 1 \leq i<j\right) \\
& =\prod_{j=1}^{d} \mathbf{E}\left(\left(1-\frac{1}{\left|L^{*}\left(w_{j}\right)\right|}\right)^{\theta_{x, j}^{*}}\right) \\
& \geq \prod_{j=1}^{d}\left(1-\frac{1}{\left|L\left(w_{j}\right)\right|-\gamma \delta^{-1}}\right)^{\theta_{x, j}}
\end{aligned}
$$

since $\left|L^{*}\left(w_{j}\right)\right| \geq\left|L\left(w_{j}\right)\right|-\gamma \delta^{-1}$ and $L^{*}\left(w_{j}\right) \subseteq L\left(w_{j}\right)$ implying $\theta_{x, j}^{*} \leq \theta_{x, j}$.

Then, following [2],

$$
\begin{aligned}
\mathbf{E}\left(Z_{\sigma}\right) & \geq \sum_{x \in[q]} \prod_{j=1}^{d}\left(1-\frac{1}{\left|L\left(w_{j}\right)\right|-\gamma \delta^{-1}}\right)^{\theta_{x, j}} \\
& \geq q\left(\prod_{x \in[q]} \prod_{j=1}^{d}\left(1-\frac{1}{\left|L\left(w_{j}\right)\right|-\gamma \delta^{-1}}\right)^{\theta_{x, j}}\right)^{1 / q} \\
& =q\left(\prod_{j=1}^{d}\left(1-\frac{1}{\left|L\left(w_{j}\right)\right|-\gamma \delta^{-1}}\right)^{\left|L\left(w_{j}\right)\right|}\right)^{1 / q} \\
& \geq q \exp \left\{-\frac{1}{q} \sum_{j=1}^{d} \frac{\left|L\left(w_{j}\right)\right|}{\left|L\left(w_{j}\right)\right|-1-\gamma \delta^{-1}}\right\}, \quad \text { using } 1-x \geq e^{-x /(1-x)} \text { for } 0<x<1, \\
& \geq q \exp \left\{-\frac{\Delta}{q} \cdot \frac{q-\Delta}{q-\Delta-1-\gamma \delta^{-1}}\right\} \\
& \geq\left(1+\frac{\epsilon}{2}\right) \Delta .
\end{aligned}
$$

(If $f(x)=x e^{-1 / x}$ then $f(\alpha)=1$ and $f^{\prime}(\alpha) \sim .891$.)

We will now prove that for all $\sigma \in \Omega, Z_{\sigma}$ is concentrated around its mean via the use of the AzumaHoeffding martingale inequality. To this end, let $x_{1}, x_{2}, \ldots, x_{d}$ be the colours assigned to $w_{1}, w_{2}, \ldots, w_{d}$. Thus we can write $Z_{\sigma}=Z_{\sigma}\left(x_{1}, x_{2}, \ldots, x_{d}\right)$. Now let

$$
Z_{\sigma, i}=Z_{\sigma, i}\left(x_{1}, x_{2}, \ldots, x_{i}\right)=\mathbf{E}\left(Z \mid x_{1}, x_{2}, \ldots, x_{i}\right) .
$$

We will show next that for all feasible colours $x_{1}, x_{2}, \ldots, x_{i}, x_{i}^{\prime}$ that

$$
\left|Z_{\sigma, i}\left(x_{1}, x_{2}, \ldots, x_{i-1}, x_{i}\right)-Z_{\sigma, i}\left(x_{1}, x_{2}, \ldots, x_{i-1}, x_{i}^{*}\right)\right| \leq 2
$$


The aforementioned inequality will then imply that for any $t \geq 0$,

$$
\operatorname{Pr}\left(Z_{\sigma}-\mathbf{E}\left(Z_{\sigma}\right) \leq-t\right) \leq e^{-t^{2} /(2 d)}
$$

and then taking $t=\epsilon \Delta / 4$ and using (6) we get

$$
\operatorname{Pr}\left(Z_{\sigma} \leq\left(1+\frac{\epsilon}{4}\right) \Delta\right) \leq e^{-\epsilon^{2} \Delta / 32} .
$$

This together with Lemma 3.1 and (4) implies (2).

To prove (7), fix $i, x_{1}, x_{2}, \ldots, x_{i}, x_{i}^{*}$. In one instance of $\mathcal{P}_{\sigma}$ we start by colouring $w_{1}, w_{2}, \ldots, w_{i}$ with $x_{1}, x_{2}, \ldots, x_{i}$ to produce colouring $\tau$. In another instance we start by colouring $w_{1}, w_{2}, \ldots, w_{i}$ with $x_{1}, x_{2}, \ldots, x_{i}^{*}$ to produce colouring $\tau^{*}$.

We couple these two constructions in order to minimise the expected difference in the number of vertices $U$ with a different colour. A paths of disagreement argument gives that

$$
\mathbf{E}(U) \leq 1+\sum_{j=i+1}^{d}\left(\frac{\gamma \delta^{-1}}{\left|L\left(w_{j}\right)\right|-\gamma \delta^{-1}}\right)^{j-i} \leq 2
$$

and (7) follows.

Explanation of (8): We claim that if $c_{j}, c_{j}^{*}$ is the colour of $v_{j}$ in $\sigma_{d}, \sigma_{d}^{*}$ respectively, then

$$
\operatorname{Pr}\left(c_{j} \neq c_{j}^{*}\right) \leq\left(\frac{\gamma \delta^{-1}}{\left|L\left(w_{j}\right)\right|-\gamma \delta^{-1}}\right)^{j-i} .
$$

This is because if $c_{j} \neq c_{j}^{*}$ then there is a path of disagreements $v_{i_{1}}, v_{i_{2}}, \ldots, v_{i_{s}}$ where $i=i_{1}<i_{2}<$ $\cdots<i_{s}=j$ such that $c_{i_{r}} \neq c_{i_{r}}^{*}$ for $1 \leq r \leq s$. There are at most $\left(\lambda \delta^{-1}\right)^{j-i}$ such paths and each has probability at most $\left(\left|L\left(w_{j}\right)\right|-\gamma \delta^{-1}\right)^{i-j}$ of all vertices being coloured differently. 


\section{References}

[1] N. Alon, M. krivelevich and B. Sudakov, Coloring graphs with sparse neighborhoods, Journal of Combinatorial Theory, Ser. B 77 (1999) 73-82.

[2] M. Dyer, A. Frieze, Randomly colouring graphs with lower bounds on girth and maximum degree, Random Structures and Algorithms, 23 167-179, 2003.

[3] M.E. Dyer, A.M. Frieze and R. Kannan, A random polynomial time algorithm for approximating the volume of convex bodies, Journal of the Association for Computing Machinery 38(1):1-17, 1991.

[4] M.E. Dyer, A. Flaxman, A.M. Frieze and E. Vigoda, Randomly colouring sparse random graphs.

[5] M.E. Dyer, A.M. Frieze, T. P. Hayes and E. Vigoda, Randomly coloring constant degree graphs.

[6] L. Goldberg, R. Martin and M. Paterson, Strong spatial mixing for graphs with fewer colours.

[7] T. P. Hayes, Randomly coloring graphs of girth at least five, Proceedings of the 35th Annual ACM Symposium on Theory of Computing, (2003) 269-278

[8] T. P. Hayes and E. Vigoda, A Non-Markovian Coupling for Randomly Sampling Colorings. Proceedings of the 44th Annual IEEE Symposium on Foundations of Computer Science, (2003) 618-627.

[9] T. P. Hayes and E. Vigoda, Coupling with the stationarity distribution and improved sampling fror colorings and independent sets.

[10] M.R. Jerrum, A very simple algorithm for estimating the number of $k$-colourings of a low-degree graph, Random Structures and Algorithms 7 (1995), 157-165.

[11] M.R. Jerrum, Counting, sampling and integrating: algorithms and complexity, Lectures in Mathematics-ETH Zürich, Birkhäuser, to appear January 2003.

[12] M.R. Jerrum, A. Sinclair and E. Vigoda, A polynomial-time approximation algorithm for the permanent of a matrix with non-negative entries, Proceedings of the 12th Annual ACM-SIAM Symposium on Discrete Algorithms (2001), 712-721.

[13] M.R. Jerrum, L.G. Valiant and V.V. Vazirani, Random generation of combinatorial structures from a uniform distribution, Theoretical Computer Science 43 (1986), 169-188.

[14] A. Johansson, Asymptotic choice number for triangle-free graphs, DIMACS technical report 1996.

[15] R. Kannan, L. Lovász and M. Simonovits, Random walks and an $O^{*}\left(n^{5}\right)$ volume algorithm for convex bodies, Random Structures and Algorithms 11 (1997), 1-50.

[16] L. Lovász and S. Vempala, Simulated annealing in convex bodies and an $O^{*}\left(n^{4}\right)$ volume algorithm, to appear in JCSS.

[17] M. Molloy, The Glauber dynamics on colorings of a graph with high girth and maximum degree. To appear in SIAM Journal on Computing.

[18] M. Molloy and B. Reed, Graph Colouring and the Probabilistic Method, Springer, 2002.

[19] J. van den Berg and J.E. Steif, Percolation and the hard-core lattice gas model, Stochastic Processes and their Applications 49 (1994), 179-197.

[20] E. Vigoda, Improved bounds for sampling colorings, Proceedings of the 40th Annual IEEE Symposium on Foundations of Computer Science (1999), 51-59.

[21] V. Vu, An upper bound on the list chromatic number of locally sparse graphs, Combinatorics, Probability and Computing 11 (2002), 103-111 
